\title{
ON THE RESOLUTION OF THE SENSITIVITY CONJECTURE
}

\author{
ROHAN KARTHIKEYAN, SIDDHARTH SINHA, AND VALLABH PATIL
}

\begin{abstract}
The sensitivity conjecture is a long-standing problem in theoretical computer science that seeks to fit the sensitivity of a Boolean function into a unified framework formed by the other complexity measures of Boolean functions, such as block sensitivity and certificate complexity. After more than thirty years of attacks on this conjecture, Hao Huang (2019) gave a very succinct proof of the conjecture. In this survey, we explore the ideas that inspired the proof of this conjecture by an exposition of four papers that had the most impact on the conjecture. We also discuss progress on further research directions that the conjecture leads us to.
\end{abstract}

\section{Contents}

1. Introduction

2. Some complexity measures of Boolean functions

3. Chung et al.'s result

3.1. Existence of a subgraph with maximum degree $\lceil\sqrt{n}\rceil$

3.2. A logarithmic lower bound for $\Delta(G)$

4. Nisan and Szegedy's result

4.1. Some Fourier analysis

4.2. A logarithmic lower bound for $\operatorname{deg}(f)$

4.3. Two inequalities concerning $\operatorname{deg}(f), \widetilde{\operatorname{deg}}(f)$ and $D(f)$

5. Gotsman and Linial's result

5.1. Reformulating the sensitivity conjecture

5.2. Consequences

6. Hao's result

6.1. Cauchy's interlace theorem

6.2. Proving the sensitivity conjecture

6.3. Constructing the matrix

7. Further discussion

7.1. Three notions of symmetry 634

7.2. Problems

8. Sensitivity and CREW PRAMs

Acknowledgments

About the authors $\quad 636$

References 637

Received by the editors January 8, 2020.

2010 Mathematics Subject Classification. Primary 68R10, 05C35, 05-02; Secondary 68Q17, 94C10, 41A10, 42A16, 05E05, 15A24. 


\section{INTRODUCTION}

Boolean functions map a sequence of $n$ bits to a single bit vector 0 or 1 , represented as False and True, respectively. Some of the simplest Boolean functions are the AND function $x \cdot y$, the OR function (nonexclusive) $x+y$, and the NOT function $\bar{x}=1-x$. An important problem in Boolean function complexity- the circuit lower bound problem - is

How many of these simplest functions do we need to calculate the value of a given Boolean function on all input vectors?

The main challenge lies in the nature of the circuit, where small circuits may work counterintuitively, thereby making it difficult to ascertain the answer quickly. Nevertheless, the answer to the above question is called the circuit complexity of the Boolean function $f$.

There are several useful measures to describe the complexity of a Boolean function that can be stated mathematically. Two such measures are sensitivity and block sensitivity.

Definition 1.1. The sensitivity, $s(f)$, of a Boolean function $f:\{0,1\}^{n} \rightarrow\{0,1\}$ on $n$ variables is defined to be the largest number for which there is an $x$ such that there are at least $s(f)$ values of $i=1, \ldots, n$ with $f\left(x+e_{i}\right) \neq f(x)$. Here $e_{i}$ is the Boolean vector with exactly one 1 in the $i$ th position.

Definition 1.2. The block sensitivity, $b s(f)$, is defined to be the maximum number of disjoint subsets of $B_{1}, \ldots, B_{t}$ of $[n]=\{1, \ldots, n\}$ such that for all $1 \leq$ $j \leq t, f(x) \neq f\left(x^{B_{j}}\right)$, where $x^{B_{j}}$ is the Boolean string which differs from $x$ on exactly the bits of $B_{j}$.

Block sensitivity is known to be polynomially related to a number of other complexity measures of $f$, including the decision-tree complexity and the certificate complexity (see section 2 for definitions). A long-standing open question that existed was whether sensitivity also belonged to this equivalence class. A positive answer to this question is commonly known as the sensitivity conjecture first proposed by Nisan and Szegedy [3].

Conjecture 1.3 (Sensitivity Conjecture). There exists an absolute constant $C>0$, such that for every Boolean function $f$,

$$
b s(f) \leq s(f)^{C} .
$$

It may be useful to think of the sensitivity of a Boolean function in the following way: Take an array of $n$ switches, with some wiring for a single light bulb. For different configurations of switches flipped on or off, the light bulb is either on or off. Then the circuit is said to be sensitive, with respect to the $i$ th switch if, for some configuration of the states of the switches, flipping the $i$ th switch changes the state of the light bulb from on to off, or vice versa. For that configuration, there may be more than one switch for which the circuit is sensitive. If we count the number of such switches at which the circuit is sensitive and do so for every configuration of the switches, then the greatest number of switches for which the circuit was sensitive for some configuration, is the sensitivity $s(f)$, where $f$ is the Boolean function (ON/OFF) expressed by the circuit.

The sensitivity conjecture can also be seen as concerning whether sensitivity is polynomially related to $n$, the number of input variables to the Boolean function. 
To resolve this, Gotsman and Linial [1] proved the equivalence between the following two problems:

(1) Denote the $n$-dimensional cube by $Q_{n}=\{-1,1\}^{n}$ and the maximal degree of any graph $G$ by $\Delta(G)$. For an induced subgraph $G$ of $Q_{n}$ with strictly greater than half the number of vertices, i.e., greater than $2^{n-1}$ vertices, find an effective lower bound for $\Delta(G)$ in terms of $n$.

(2) Let $f: Q_{n} \rightarrow\{-1,1\}$ be a Boolean function, with sensitivity $s(f)$. Denoting the degree of the multilinear polynomial representation of $f(x)$ by $\operatorname{deg}(f)$, find an effective upper bound for $\operatorname{deg}(f)$ in terms of $s(f)$.

It was previously established by Chung, Furedi, Graham, and Seymour 2 that for an induced subgraph $G$ of $Q_{n}$ with strictly more than half its vertices, the degree of some vertex $v$ in $G$ is bounded above logarithmically in $n$ (see section 3.2). Hao 44 recently improved this logarithmic bound to a polynomial relation.

Theorem 1.4 (Hao). For every integer $n \geq 1$, let $G$ be an arbitrary $\left(2^{n-1}+1\right)$ vertex induced subgraph of $Q_{n}$. Then

$$
\Delta(G) \geq \sqrt{n}
$$

This result proves that the sensitivity and degree of a Boolean function are polynomially related, thereby confirming the sensitivity conjecture (see section 6.2).

In the past three decades, a great amount of research has been done on resolving the sensitivity conjecture, resulting in a wide-ranging body of knowledge. In this survey, we could not hope to cover even a fraction of it. Our goal behind the selection of this material has largely been to portray the historical development of the results that have had the most impact on the development of the conjecture. We would like to direct the attention of the interested reader to the surveys of Buhrman and de Wolf [9], and Hatami, Kulkarni, and Pankratov [10] for more in-depth discussions on the conjecture.

After defining complexity measures of Boolean functions, we look at Chung et al.'s 2] result of finding the maximum degree of an induced subgraph of the cube graph $Q_{n}$ with strictly more than half its vertices. In a different direction, we look at how Nisan and Szegedy [3] proposed the sensitivity conjecture which was reduced to a question of finding a polynomial bound for the maximum degree of an induced subgraph of $Q_{n}$ by Gotsman and Linial [1]. We then explain how Hao 4. proved this graph-theoretic question thereby positively resolving the sensitivity conjecture, before finishing with recent progress on some open questions of Hao and an application of the sensitivity conjecture to CREW PRAMs.

\section{Some COMPlexity measures of Boolean functions}

Consider the set $Q_{n}=\{-1,1\}^{n}$. The hypercube of dimension $n$ is the graph with vertex set $Q_{n}$ and an edge between $x=\left(x_{1}, x_{2}, \ldots, x_{n}\right)$ and $y=\left(y_{1}, y_{2}, \ldots, y_{n}\right)$ in $Q_{n}$ if there is exactly one $i$ such that $x_{i} \neq y_{i}$. Let $f$ be a function mapping $Q_{n}$ to $\{-1,1\}$.

Definition 2.1. The sensitivity of $f$ at $x$, denoted $s(f, x)$, is defined as the number of neighbors $y$ of $x$ for which $f(x) \neq f(y)$, that is, the number of $i$ such that

$$
f\left(x_{1}, \ldots, x_{i}, \ldots, x_{n}\right) \neq f\left(x_{1}, \ldots,-x_{i}, \ldots, x_{n}\right) .
$$




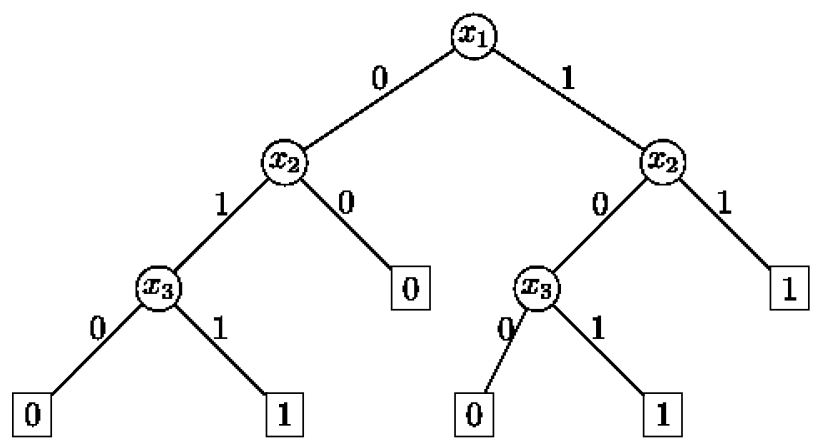

FIGURE 1. Decision tree of depth 3 computing majority on three variables

This local measure can be naturally extended to yield the value of the (global) sensitivity of $f$, which we defined in Definition 1.1. A natural generalization of sensitivity is to flip blocks of bits rather than single bits. This leads us to the complexity measure of block sensitivity of $f$, defined in Definition 1.2 .

In the decision-tree model, we compute the value of a given function at an unknown input. Considering Boolean functions, we collect information about the input by asking questions of the form, "What is the value of input $x_{i}$ ?" Each question asked depends only on the information gained so far.

A (deterministic) decision tree can then be defined as a rooted binary tree with labels on each node and edge. Each inner node is labeled by a query. One of the two edges leaving the node is labeled 0 , the other is labeled 1 . The two labels represent the two possible answers to the query. Each leaf, labeled 0 or 1 , gives the output (Figure 11). The Boolean function computed by the given decision tree takes the label at this leaf as the value on the given input.

Define $\operatorname{cost}(A, x)$ as the number of queries asked when the decision tree $A$ is executed on input $x$. That is, it is the length of the computation path forced by $x$. Also, $\max _{x} \operatorname{cost}(A, x)$ defines the worst case complexity of $A$, that is, the tree's depth.

Definition 2.2. The decision tree complexity of $f$, denoted by $D(f)$, is given by

$$
D(f)=\min _{A} \max _{x} \operatorname{cost}(A, x) .
$$

That is, it is the depth of the minimum-depth decision tree that computes $f$. It is obvious that any function $f:\{0,1\}^{n} \rightarrow\{0,1\}$ can be computed by asking $n$ questions.

Take a vector $x \in\{0,1\}^{n}$. A $b$-certificate (for $b \in\{0,1\}$ ) is a subset $S \subseteq[n]$ such that $f(y)=f(x)$ for all vectors $y \in\{0,1\}^{n}$ such that $y_{i}=x_{i}$ for all $i \in S$.

Definition 2.3. The certificate complexity of a Boolean function $f$ on $x$, denoted $C_{x}(f)$, is the minimum size of a $b$-certificate for $x$.

The certificate complexity of $f$, denoted $C(f)$, is defined as

$$
C(f)=\max _{x \in Q_{n}} C_{x}(f) .
$$

To visualize this, one can think of a Boolean function as coloring the vertex on a $n$-dimensional hypercube using just two colors. Then, the certificate complexity is $n$ minus the dimension of the largest monochromatic hypercube in $Q_{n}$. 


\section{Chung et Al.'s Result}

In this section, we expound Chung et al.'s result [2]: an induced subgraph of the $n$-dimensional cube graph $Q_{n}$ with strictly more than half of its vertices has a maximum degree of at least $o(\log n)$. We will also look at the construction of a $\left(2^{n-1}+1\right)$-vertex induced subgraph whose maximum degree is $\lceil\sqrt{n}\rceil$.

Definition 3.1. Let $G=(V, E)$ be a graph. For $S \subset V$, an induced subgraph $G[S]$ is the graph with vertex set $S$ and edge set $E^{\prime}$, where $E^{\prime}$ consists of all edges in $E$ that have both endpoints in $S$.

Definition 3.2. The maximum degree $\Delta(G)$ of a graph $G=(V, E)$ is defined as

$$
\Delta(G)=\max _{v \in V(G)} \operatorname{deg}_{G}(v)
$$

In section 3.1, we construct a subgraph $G$ of $Q_{n}$ with $\Delta(G)<\sqrt{n}+1$, proving (2); in section 3.2. we show the logarithmic lower bound for $\operatorname{deg}_{G}(v)$ given by (11):

Theorem 3.3. Let $G$ be an induced subgraph of $Q_{n}$ with at least $2^{n-1}+1$ vertices. Then for some vertex $v$ of $G$, we have

$$
\operatorname{deg}_{G}(v)>\frac{1}{2} \log n-\frac{1}{2} \log \log n+\frac{1}{2} .
$$

Moreover, there exists a $\left(2^{n-1}+1\right)$-vertex induced subgraph $G$ of $Q_{n}$ with

$$
\Delta(G)<\sqrt{n}+1 .
$$

3.1. Existence of a subgraph with maximum degree $\lceil\sqrt{n}\rceil$. Denote that $\{1,2, \ldots, n\}:=[n]$. Consider a family of sets $2^{[n]}$, that is, the set of all subsets of $[n]$. Observe that the natural bijection between the set of $n$-bit vectors $\{0,1\}^{n}$ and $2^{[n]}$ is the map $\psi:\{0,1\}^{n} \leftrightarrow 2^{[n]}$ such that the vector $\mathbf{x}=\left(x_{1}, x_{2}, \ldots, x_{n}\right)$ is mapped to $T=\left\{i \mid x_{i}=1,1 \leq i \leq n\right\}$, and vice versa.

Extending the bijection between the $n$-bit vectors and $2^{[n]}$, partition $Q_{n}$ into two vertex-disjoint subgraphs $G$ and $G^{\prime}$ where $G$ has $2^{n-1}+1$ vertices. Define the family of sets $\mathbf{X}$ over a finite collection of subsets $\mathbf{F} \subset 2^{[n]}$ as

$$
\begin{aligned}
\mathbf{X}(\mathbf{F})= & \{S \subset[n]:|S|=\text { even, } \exists F \in \mathbf{F} \text { with } F \subset S\} \\
& \cup\{S \subset[n]:|S|=\text { odd }, F \backslash S \neq \phi \text { for all } F \in \mathbf{F}\} .
\end{aligned}
$$

That is, $\mathbf{X}(\mathbf{F})$ is the collection of all even sets which contain some $F \in \mathbf{F}$ along with all odd sets which contain no $F \in \mathbf{F}$.

Consider $\mathbf{F}$ as the partition of $[n]$ given by $[n]=F_{1} \cup F_{2} \cup \cdots \cup F_{k}$ such that $|k-\sqrt{n}|<1$ and ||$F_{i}|-\sqrt{n}|<1,1 \leq i \leq k$. We prove the existence of such a partition:

Proof. Let $k=\lceil\sqrt{n}\rceil \Longrightarrow|k-\sqrt{n}|<1$. Denote $u:=\sqrt{n} \Longrightarrow u^{2}=n$. It is easy to see that there exists an $\epsilon$ with $0 \leq \epsilon<1$ so that $\lfloor\sqrt{n}\rfloor=\sqrt{n}-\epsilon=u-\epsilon$ and $\lceil\sqrt{n}\rceil=\sqrt{n}-\epsilon+1=u-\epsilon+1$.

For the above partition to exist, we need to show that $\exists x, y \in \mathbb{Z}^{+}$such that $x\lfloor\sqrt{n}\rfloor+y\lceil\sqrt{n}\rceil=n$ with $x+y=\lceil\sqrt{n}\rceil=u-\epsilon+1$. Note that

$$
x(u-\epsilon)+y(u-\epsilon+1)=u^{2}
$$


must have integer solutions. Observe that

$$
\begin{aligned}
(3) & \Longrightarrow x(x+y-1)+y(x+y)=u^{2} \\
& \Longrightarrow x^{2}+y^{2}+2 x y-x=u^{2} \\
& \Longrightarrow(x+y)^{2}=u^{2}+x .
\end{aligned}
$$

Clearly, this has integer solutions as we may fix $u^{2}$ and $x$ and so vary $y$ to get a set of solutions.

For such a partition of $[n]$, we wish to see if the following claims are true.

Claim 3.4. $|\mathbf{X}|=2^{n-1} \pm 1$, according to whether $n+k$ is odd or even.

Claim 3.5. $\Delta \leq k$ for the subgraph induced by $\mathbf{X}(\mathbf{F})$ and $2^{[n]} \backslash \mathbf{X}(\mathbf{F})$.

Define the rank of $\mathbf{F}$, denoted $r(\mathbf{F})$, as the largest size of an element in $\mathbf{F}$. Denote $t(\mathbf{F})$ to be the largest size of the disjointly representable subsystems of $\mathbf{F}$. That is, $t(\mathbf{F})$ is the maximum value of $t$ such that one can find $F_{1}, F_{2}, \ldots, F_{t} \in \mathbf{F}$ and $x_{i} \in F_{i}, 1 \leq i \leq t$, such that $x_{i} \in F_{j} \leftrightarrow i=j$, for all $1 \leq i, j \leq k$, that is, no $F_{i}$ is contained in the union of the others.

In the partition chosen for $\mathbf{F}$ such that we had $k$ disjointly representable subsets of $\mathbf{F}$, note that $t(\mathbf{F})=k$ and $r(\mathbf{F})$ is either $\lfloor\sqrt{n}\rfloor$ or $\lceil\sqrt{n}\rceil$. The maximum degree $\Delta(G(\mathbf{F}))$ is either bounded by $r(\mathbf{F})$ or $t(\mathbf{F})$, since the degree of a vertex corresponding to some $S \in \mathbf{X}(\mathbf{F})$ is the number of $S_{i} \in \mathbf{X}(\mathbf{F})$ such that $S$ and $S_{i}$ differ by only one element. We can then easily see that $\Delta(G(\mathbf{F}))<\sqrt{n}$.

Lemma 3.6. $\Delta(G(\mathbf{F})) \leq \max \{r(\mathbf{F}), t(\mathbf{F})\}$.

Remark 3.7. The same result also holds true for $\Delta\left(G^{\prime}(\mathbf{F})\right)$.

Proof. An interesting thing to note is that this result holds for any family of subsets of $2^{[n]}$. To see this, consider the sets corresponding to the vertices (1 10111$)$, $(11010),(01011)$ of $G(\mathbf{F})$ for some $\mathbf{F} \subset 2^{[n]}$. The subsets $\{1,2,4\}$ and $\{2,4,5\}$ belong to $\mathbf{X}(\mathbf{F})$ so long as no subset of either belongs to $\mathbf{F}$. Therefore, there are no even subsets in $\mathbf{F}$ which are contained in odd sets of $\mathbf{X}(\mathbf{F})$.

Consequently, (11111) cannot belong to $G(\mathbf{F})$ as it would contain some $F \in \mathbf{F}$. Consider again the vertex (1 1011 ). It can have maximum degree 4 (in the 5 -cube

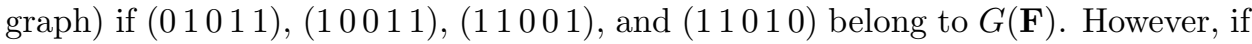
no subsets of the vertices adjacent to $\left(\begin{array}{lllll}1 & 1 & 0 & 1 & 1\end{array}\right)$ belong to $\mathbf{F}$, then only the singleton

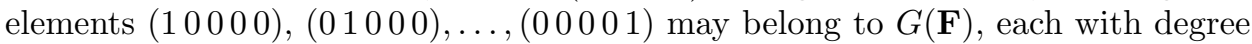
at most 1 .

Therefore, for any edge of $Q_{n}$ corresponding to $\left(S, S^{\prime}\right)$, where $S, S^{\prime} \in \mathbf{X}(\mathbf{F})$ and $S$ is even, the odd set $S^{\prime}$ is a proper subset of $S$. Since, $S$ and $S^{\prime}$ differ by the inclusion of precisely one element of $[n]$, and the exclusion of that element from $S$ results in a subset not contained in $\mathbf{F}$, then

$$
\operatorname{deg}(S) \leq|\bigcap\{F: F \in \mathbf{F}, F \subset S\}| \leq r(\mathbf{F}) .
$$

Taking $S$ as the odd set, we instead get $S \subset S^{\prime}$, and by the construction of $G(\mathbf{F})$, there exists a $F \in \mathbf{F}$ such that $F \subset S^{\prime}$ and $F \not \subset S$. Suppose, we have the sets $S_{1}^{\prime}, S_{2}^{\prime}, \ldots, S_{a}^{\prime}$ such that there is an edge between $S$ and $S_{i}^{\prime}, 1 \leq i \leq a$. Then, there must also be some sets $F_{1}, F_{2}, \ldots, F_{a} \in \mathbf{F}$ which do not belong to $S$, but $F_{i} \in S_{i}^{\prime}$. Since, $S$ and $S_{i}^{\prime}$ differ by the inclusion of exactly one element, $F_{1}, F_{2}, \ldots, F_{a}$ must 
be disjointly representable. Hence, $a \leq t(\mathbf{F})$. Since the quantity $a$ gives the number of adjacent vertices, this proves the lemma.

The cardinality of $\mathbf{X}(\mathbf{F})$ can be found using the principle of inclusion-exclusion (also called the sieve formula) and is given as

$$
|\mathbf{X}(\mathbf{F})|=2^{n-1}+(1-2 \epsilon)\left[\sum_{\substack{F_{i} \in \mathbf{F} \\\left|F_{i}\right|=n}} 1-\sum_{\substack{F_{i} \in \mathbf{F} \\\left|F_{i} \cup F_{j}\right|=n}} \sum_{F_{j} \in \mathbf{F}} 1+\cdots\right],
$$

where $\epsilon=0$ when $n$ is even and $\epsilon=1$ when odd.

Denote by $f(\mathbf{F})$ the bracketed expression on the right-hand side of (5). Taking $\mathbf{F}$ as the partition of $[n]$ into $F_{1}, F_{2}, \ldots, F_{k}, f(\mathbf{F})$ equals $(-1)^{k+1}$. And, because $(1-2 \epsilon)=(-1)^{n}$ we have

$$
|\mathbf{X}(\mathbf{F})|=2^{n-1}+(-1)^{n+k+1},
$$

thus proving Claim 3.4 .

Now, to prove Claim 3.5

Lemma 3.8. Suppose $f(\mathbf{F}) \neq 0$. Then, $\max \{r(\mathbf{F}), t(\mathbf{F})\} \geq \sqrt{n}$.

Remark 3.9. We choose the maximum of $r(\mathbf{F})$ and $t(\mathbf{F})$ since the degree is bounded by both values, and the greater value is the one that dominates the bound.

Proof. Note that there are $2^{n-1}$ possible $n$-bit vectors such that every one of them has their $j$ th component as 0 . Since, we are taking $2^{n-1}+1$ vertices, by the pigeonhole argument, there is at least one vector in every direction, that is, has a nonzero value for the $j$ th component. This provides the motivation for taking $f(\mathbf{F}) \neq 0$. Since vertices of $G(\mathbf{F})$ correspond to the subsets of $2^{[n]}$, this implies that $|\bigcup \mathbf{F}|=n$.

Choose a subfamily $\left\{F_{1}, F_{2}, \ldots, F_{s}\right\}$ of $\mathbf{F}$ such that this family has the smallest number of sets with $\bigcup_{i=1}^{s} F_{i}=[n]$. Now, $r(\mathbf{F})<\sqrt{n}$, else the inequality $\max \{r(\mathbf{F}), t(\mathbf{F})\} \geq \sqrt{n}$ is trivial for $|F| \geq \sqrt{n}$. Then, we must check if $t(\mathbf{F}) \geq \sqrt{n}$ in such a case. Since, $s$ is the minimum number such that $\bigcup_{i=1}^{s} F_{i}=[n]$, so $\left\{F_{1}, F_{2}, \ldots, F_{s}\right\}$ must be disjointly representable, else there would be a contradiction to the minimality of $s$.

Since $\left|F_{i}\right|$ cannot exceed $\sqrt{n}$, observe that if $\max \left|F_{i}\right|<\sqrt{n}$, and if $s<\sqrt{n}$, then $s \cdot \max \left\{\left|F_{i}\right|\right\}<|[n]|=n$, which is a contradiction, since $\bigcup_{i=1}^{s} F_{i}=[n]$. Therefore $s \geq \sqrt{n}$, and so

$$
\sqrt{n} \leq \max \{r(\mathbf{F}), t(\mathbf{F})\} .
$$

Since $\sqrt{n}<n$ for $n \geq 1$, the maximal degree $\Delta(G) \leq \sqrt{n}$, and since both $\Delta(G)$ and $\sqrt{n}$ are bounded above by $\max \{r(\mathbf{F}), t(\mathbf{F})\}$, we can have a partition $G(\mathbf{F})$ such that $\Delta(G) \ll \sqrt{n}$. This proves (2).

\subsection{A logarithmic lower bound for $\Delta(G)$.}

Lemma 3.10. Let $G$ be a subgraph of $Q_{n}$ with average degree $\bar{d}$. Then, $|V(G)| \geq 2^{\bar{d}}$.

Proof. Let us use induction on $|V(G)|$. Split $Q_{n}$ into two $(n-1)$-dimensional subcubes $Q_{1}$ and $Q_{2}$ such that $V_{1}=Q_{1} \cap V(G) \neq \phi$ and $V_{2}=Q_{2} \cap V(G) \neq \phi$. Also, assume that $\left|V_{2}\right| \geq\left|V_{1}\right|$ and that there are $s$ edges between $V_{1}$ and $V_{2}$ in $G$. Because no more than one edge can come out of any vertex in $V_{1}$, this would mean 
that $\left|V_{1}\right| \geq s$. Let the restriction of $G$ to $V_{i}(i=1,2)$ be denoted by $G_{i}$. Then, the induction hypothesis gives us

$$
\begin{gathered}
\left|V_{i}\right| \log _{2}\left|V_{i}\right| \geq \sum \operatorname{deg}_{G_{i}}(v)=\sum_{v \in V_{i}} \operatorname{deg}_{G}(v)-s \\
\Longrightarrow\left|V_{1}\right| \log _{2}\left|V_{1}\right|+\left|V_{2}\right| \log _{2}\left|V_{2}\right|+2 s \geq \sum_{v \in V(G)} \operatorname{deg}_{G}(v) .
\end{gathered}
$$

Using $\log _{2}$ arithmetic and the fact that $\left|V_{2}\right| \geq\left|V_{1}\right|$

$$
\left(\left|V_{1}\right|+\left|V_{2}\right|\right) \log _{2}\left(\left|V_{1}\right|+\left|V_{2}\right|\right) \geq\left|V_{1}\right| \log _{2}\left|V_{1}\right|+\left|V_{2}\right| \log _{2}\left|V_{2}\right|+2\left|V_{1}\right|,
$$

thus proving the lemma.

Lemma 3.11. Suppose $G$ is a $\left(2^{n-1}\right)$-vertex induced subgraph of $Q_{n}$ containing edges from all the $n$ directions. Then, $\Delta(G)>\frac{1}{2} \log n-\frac{1}{2} \log \log n+\frac{1}{2}$.

Remark 3.12. Observe that this proves a result stronger than (11).

Proof. Let $i \in[n]$ be an arbitrary but fixed dimension. Construct three sets of vertices:

(1) $X_{i}=\left\{x \in V(G): x^{(i)} \in V(G)\right\}$, the set of endpoints of the edges of $G$ in direction $i$;

(2) $Y_{i}=\left\{y \notin V(G): y^{(i)} \notin V(G)\right\} \equiv\left\{y \in V\left(Q_{n} \backslash G\right): y^{(i)} \in V\left(Q_{n} \backslash G\right)\right\}$; and

(3) $A_{i}=V\left(Q_{n}\right) \backslash X_{i} \backslash Y_{i}$.

Let $\Delta=\Delta(G)$, and consider a pair $x, x^{(i)} \in X_{i}$.

Claim 3.13. $x$ has at least $n-2 \Delta+1$ neighbors in $Y_{i}$.

Proof. Let $y \in A_{i}$. Using the definition of $\Delta(G)$ and the fact that either one of $y$ or $y^{(i)}$ is in $V(G)$, we have the following.

(1) There are at most $\Delta-1$ neighbors of $x$ in $A_{i}$ (because we know $x$ and $x^{(i)}$ are adjacent). Let us denote them by $\left\{x^{(j)}: \#\{j\} \leq \Delta-1\right\}$.

(2) There are at most $\Delta-1$ neighbors of $x^{(i)}$ in $A_{i}$, denoted by $\left\{x^{(i j)}: \#\{j\} \leq\right.$ $\Delta-1\}$, where $x^{(i j)}$ agrees with $x$ in every dimension except $i$ and $j$.

(3) Consequently, there are at most $2(\Delta-1)$ neighbors of $x$ in $A_{i}$.

(4) There are at least $n-1-2(\Delta-1)=n-2 \Delta+1$ neighbors of $x$ in $Y_{i}$.

Observe that $|V(G)|=2^{n-1} \Longrightarrow\left|V\left(Q_{n} \backslash G\right)\right|=2^{n-1}$ and that if $G$ contains edges from all $n$ directions, then $Q_{n} \backslash G$ also contains edges from all the $n$ directions. Consequently, $\left|X_{i}\right|=\left|Y_{i}\right|$, and it is obvious that they must be greater than 0 . Using these facts, we obtain

$$
\left|E\left(G\left(X_{i} \cup Y_{i}\right)\right)\right| \geq \frac{1}{2}\left|X_{i}\right|+\frac{1}{2}\left|Y_{i}\right|+(n-2 \Delta+1)\left|X_{i}\right|
$$

from adding the edges within the graphs $X_{i}, Y_{i}$ and also between them. After some calculations and using the fact that $\left|X_{i}\right|=\left|Y_{i}\right|$, we get

$$
\bar{d}\left(G\left(X_{i} \cup Y_{i}\right)\right) \geq n-2 \Delta+2,
$$

which when used with Lemma 3.10 yields $\left|X_{i}\right| \geq 2^{n-2 \Delta+1}$.

Thus, there are at least $2^{n-2 \Delta+1}$ edges in direction $i$ in the graph $G$. Summing over all dimensions, we observe that at least $n \cdot 2^{n-2 \Delta+1}$ edges are in the graph $G$. 
On the other hand, this number cannot exceed $\Delta \cdot 2^{n-1}$. Since $n \cdot 2^{n-2 \Delta+1} \leq \Delta \cdot 2^{n-1}$, a straightforward computation shows that

$$
\Delta(G) \geq \frac{1}{2} \log n-\frac{1}{2} \log \log n+\frac{1}{2} .
$$

This proves (1).

\section{NisAn AND Szegedy's RESUlT}

In this section, we will look at Nisan and Szegedy's results [3] that formulate the sensitivity conjecture. This is done by establishing a polynomial relation between the decision-tree complexity of a Boolean function $f$, the degree of the multilinear polynomial representing $f$, and the smallest degree of the polynomial approximating $f$.

A multilinear polynomial over variables $x_{1}, \ldots, x_{n}$ has $2^{n}$ terms, one for each monomial $\prod_{i \in S} x_{i}$, where $S \subseteq[n]$ (here $\prod_{i \in \phi} x_{i}$ denotes 1 ). Observe that it has no variables squared, cubed, etc. If we define a Boolean function $f$ as $f:\{F, T\}^{n} \rightarrow$ $\{F, T\}$, then we can encode $T$ as 1 and $F$ as 0 , thus creating a mapping from a subset of $\mathbb{R}^{n}$ to a subset of $\mathbb{R}$.

Definition 4.1. A real multivariate polynomial $p: \mathbb{R}^{n} \rightarrow \mathbb{R}$ represents $f$ if for all $x \in\{0,1\}^{n}, f(x)=p(x)$.

Definition 4.2. The degree of a Boolean function $f$, denoted by $\operatorname{deg}(f)$, is the degree of the unique multilinear real polynomial that represents $f$ exactly.

Definition 4.3. The polynomial $p$ approximates $f$ if for every $x \in\{0,1\}^{n}$, we have that

$$
|p(x)-f(x)|<\frac{1}{3} .
$$

The approximate degree of $f$, that is, $\widetilde{\operatorname{deg}}(f)$ is defined to be the minimum, over all polynomials $p$ which approximate $f$, of the degree of $p$.

In section 4.2 we establish the following lower bound on the degree of a Boolean function $f$ in terms of the number of variables $n$.

Theorem 4.4. Let $f$ be a Boolean function that depends on $n$ variables. Then,

$$
\operatorname{deg}(f) \geq \log _{2} n-O(\log \log n) .
$$

In section 4.3 we prove the following inequalities:

Theorem 4.5. For every Boolean function $f$,

$$
\operatorname{deg}(f) \leq D(f) \leq 16 \operatorname{deg}(f)^{8} .
$$

Theorem 4.6. There exists a constant $c$ such that for every Boolean function, we have

$$
\widetilde{\operatorname{deg}}(f) \leq \operatorname{deg}(f) \leq D(f) \leq c \cdot \widetilde{\operatorname{deg}}(f)^{8},
$$

where $D(f)$ is the decision tree complexity of $f$ (see Definition 2.2). 
4.1. Some Fourier analysis. In this subsection, we state some results needed for our discussion from [5]. Consider the Fourier transform representation, that is, viewing the Boolean function $f$ as $f:\{-1,1\}^{n} \rightarrow\{-1,1\}$. Interpret the domain $\{-1,1\}^{n}$ of $f$ as $2^{n}$ points lying in $\mathbb{R}^{n}$ and think of $f$ as giving a \pm 1 labeling to each of these points. See [5] for a method to interpolate such data points using the indicator polynomial. One obtains the following lemma.

Lemma 4.7. Every function $f:\{-1,1\}^{n} \rightarrow \mathbb{R}$ can be expressed uniquely as a multilinear polynomial,

$$
f(x)=\sum_{S \subseteq[n]} c_{S} \prod_{i \in S} x_{i}
$$

where each $c_{S}$ is a real number.

This expression (11) is precisely the Fourier expansion of $f$. It is a convention to write the coefficient $c_{S}$ as $\hat{f}(S)$ and the monomial $\prod_{i \in S} x_{i}$ as $\chi_{S}(x)$. Thus, we finally have

$$
f(x)=\sum_{S \subseteq[n]} \hat{f}(S) \chi_{S}(x) .
$$

Denote by $\mathbf{x}=\left(\mathbf{x}_{1}, \ldots, \mathbf{x}_{\mathbf{n}}\right)$ a uniformly random string from $\{-1,1\}^{n}$, where each $\mathbf{x}_{\mathbf{i}}$ is a random variable. We can generate such an $\mathbf{x}$ by choosing each bit $\mathbf{x}_{\mathbf{i}}$ independently and uniformly from $\{-1,1\}$. The result we will need is (see [5, section 2.1]):

Theorem 4.8 (Parseval). For any $f:\{-1,1\}^{n} \rightarrow \mathbb{R}$,

$$
\sum_{S \subseteq[n]} \hat{f}(S)^{2}=\mathbf{E}_{\mathbf{x}}\left[f(\mathbf{x})^{2}\right] .
$$

For Boolean functions $f:\{-1,1\}^{n} \rightarrow\{-1,1\}$, we have $f(x)^{2}=1$ for every $x$, and hence,

Corollary 4.9 (Parseval's equality). If $f:\{-1,1\}^{n} \rightarrow\{-1,1\}$, then

$$
\sum_{S \subseteq[n]} \hat{f}(S)^{2}=1 \text {. }
$$

\subsection{A logarithmic lower bound for $\operatorname{deg}(f)$.}

Definition 4.10 (Kahn, Kalai, and Linial [7). For a Boolean function $f$ on $n$ variables and a variable $x_{i}$, the influence of $x_{i}$ on $f$, denoted by $\operatorname{Inf}_{i}(f)$, is defined to be

$$
\operatorname{Inf}_{i}(f)=\operatorname{Prob}\left[f(x) \neq f\left(x^{(i)}\right)\right]
$$

where $x^{(i)}$ denotes the string $x \in\{0,1\}^{n}$ with the $i$ th bit flipped and $x$ is chosen uniformly in $\{\text { false, true }\}^{n}$.

In words, $\operatorname{Inf}_{i}(f)$ is the probability that flipping the $i$ th coordinate flips the value of the function. We observe that:

Lemma 4.11. For any Boolean function $f$ on $n$ variables, using the Fourier transform representation of $f$, we have

$$
\sum_{i=1}^{n} \operatorname{Inf}_{i}(f)=\sum_{S}|S| \hat{f}(S)^{2} .
$$


Combining this lemma with Parseval's equality, we can conclude that:

Corollary 4.12. For any Boolean function $f$,

$$
\sum_{i=1}^{n} \operatorname{Inf}_{i}(f) \leq \operatorname{deg}(f)
$$

Lemma 4.13 (Schwartz and Zippel). Let $p\left(x_{1}, \ldots, x_{n}\right)$ be a nonzero multilinear polynomial of degree $d$. If we choose $x_{1}, \ldots, x_{n}$ at random in $\{-1,1\}$, then

$$
\operatorname{Prob}\left[p\left(x_{1}, \ldots, x_{n}\right) \neq 0\right] \geq 2^{-d} .
$$

Remark 4.14. Lemma 4.13 can be proved by induction on $n$ and by writing $p$ as a linear combination of two polynomials on $\left(x_{1}, \ldots, x_{n-1}\right)$.

Proof of Theorem 4.4. For each $i$ define a function $f^{i}$ on $n-1$ variables as follows:

$$
\begin{aligned}
& f^{i}\left(x_{1}, \ldots, x_{i-1}, x_{i+1}, \ldots, x_{n}\right) \\
& \quad=f\left(x_{1}, \ldots,-1, x_{i+1}, \ldots, x_{n}\right)-f\left(x_{1}, \ldots, 1, x_{i+1}, \ldots, x_{n}\right) .
\end{aligned}
$$

From the definition of influence, it is clear that

$$
\operatorname{Inf}_{i}(f)=\operatorname{Prob}\left[f^{i}\left(x_{1}, \ldots, x_{i-1}, x_{i+1}, \ldots, x_{n}\right) \neq 0\right],
$$

where $x_{1}, \ldots, x_{i-1}, x_{i+1}, \ldots, x_{n}$ are chosen at random in $\{-1,1\}$.

Since $f$ depends on all the variables, we have that for every $i, f^{i}$ is not identically zero, and thus we can use Lemma 4.13 and conclude that for all $i, \operatorname{Inf}_{i}(f) \geq 2^{-d}$. On the other hand, from (14) it follows that $\sum_{i} \operatorname{Inf}_{i}(f) \leq d$. Combining these two bounds gives us (8).

4.3. Two inequalities concerning $\operatorname{deg}(f), \widetilde{\operatorname{deg}}(f)$ and $D(f)$. We now return to the representation of true $=1$ and false $=0$.

Definition 4.15. A polynomial $f\left(x_{1}, x_{2}, \ldots, x_{n}\right)$ is symmetric if

$$
f\left(x_{1}, \ldots, x_{n}\right)=f\left(x_{\sigma(1)}, \ldots, x_{\sigma(n)}\right)
$$

for any permutation $\sigma$ of $[n]=\{1, \ldots, n\}$.

Definition 4.16. The symmetrization of a multivariate polynomial $p: \mathbb{R}^{n} \rightarrow \mathbb{R}$ is

$$
p^{\mathrm{sym}}\left(x_{1}, \ldots, x_{n}\right)=\frac{\sum_{\sigma \in S_{n}} p\left(x_{\sigma(1)}, \ldots, x_{\sigma(n)}\right)}{n !} .
$$

Since, the number of permutations of $[n]$ is $n$ !, we get a sum of $n$ ! terms where each term is either 0 or 1 .

Example 4.17. Consider a polynomial $p:\{0,1\}^{2} \rightarrow \mathbb{R}$ given by $p\left(x_{1}, x_{2}\right)=$ $x_{1}+x_{1} x_{2}$. Then $x_{1}+x_{1} x_{2}=x_{1}\left(1+x_{2}\right)=x_{1}$. So

$$
p^{\mathrm{sym}}\left(x_{1}, x_{2}\right)=\frac{x_{1}+x_{2}}{2} .
$$

Hence, the important point to note here is that if we are only interested in inputs $x \in\{0,1\}^{n}$, then $p^{\text {sym }}$ turns out to depend only upon $x_{1}+\cdots+x_{n}$. We can thus represent it as a univariate polynomial of $x_{1}+\cdots+x_{n}$ : 
Lemma 4.18. Taking $p: \mathbb{R}^{n} \rightarrow \mathbb{R}$ to be a multivariate polynomial, then there exists a unique univariate polynomial $\tilde{p}: \mathbb{R} \rightarrow \mathbb{R}$ such that for all $x_{1}, \ldots, x_{n} \in\{0,1\}^{n}$ we have

$$
p^{\mathrm{sym}}\left(x_{1}, \ldots, x_{n}\right)=\tilde{p}\left(x_{1}+\cdots+x_{n}\right) .
$$

Moreover, $\operatorname{deg}(\tilde{p}) \leq \operatorname{deg}(p)$.

Proof. Let the degree of $p^{\text {sym }}$ be $d$. Let $P_{k}$ denote the sum of all $\left(\begin{array}{l}n \\ k\end{array}\right)$ products $\prod_{i \in S} x_{i}$ of the $|S|=k$ different variables. Since $p^{\mathrm{sym}}$ is symmetric, it can be shown by induction that it can be written as

$$
p^{\mathrm{sym}}(x)=c_{o}+c_{1} P_{1}(x)+c_{2} P_{2}(x)+\cdots+c_{d} P_{d}(x)
$$

with $c_{i} \in \mathbb{R}$. Observe that on $x \in\{0,1\}^{n}$ with $z:=x_{1}+\cdots+x_{n}$ ones, $P_{k}$ assumes the value

$$
P_{k}(x)=\left(\begin{array}{l}
z \\
k
\end{array}\right)=\frac{z(z-1) \cdots(z-k+1)}{k !},
$$

which is a polynomial of degree $k$ of $z$. Therefore the univariate polynomial $\tilde{p}(z)$, defined by

$$
\tilde{p}(z):=c_{0}+c_{1}\left(\begin{array}{l}
z \\
1
\end{array}\right)+c_{2}\left(\begin{array}{l}
z \\
2
\end{array}\right)+\cdots+c_{d}\left(\begin{array}{l}
z \\
d
\end{array}\right),
$$

has the desired property.

As $\operatorname{deg}(p)$ is bounded below by $\operatorname{deg}(\tilde{p})$, we wish to find a bound for $\operatorname{deg}(\tilde{p})$. We will therefore need to use a result from approximation theory (see, e.g., [27]).

Theorem 4.19 (Markov Inequality). Let $p: \mathbb{R} \rightarrow \mathbb{R}$ be a univariate polynomial of degree $d$ that for any real number $a_{1} \leq x \leq a_{2}$ satisfies $b_{1} \leq p(x) \leq b_{2}$. Then, for all $a_{1} \leq x \leq a_{2}$, the derivative of $p$ satisfies

$$
\left|p^{\prime}(x)\right| \leq \frac{d^{2}\left(b_{2}-b_{1}\right)}{a_{2}-a_{1}} .
$$

Because we have information on the values of $p(x)$ only for integer $x$, we need this next lemma.

Lemma 4.20. Let $p$ be a polynomial with the following properties.

(1) For any integer $0 \leq i \leq n$, we have $b_{1} \leq p(i) \leq b_{2}$.

(2) For some real $0 \leq x \leq n$, the derivative of $p$ satisfies $\left|p^{\prime}(x)\right| \geq c$.

Then

$$
\operatorname{deg}(p) \geq \sqrt{\frac{c n}{c+b_{2}-b_{1}}} .
$$

Proof. Let $c^{\prime}=\max _{0 \leq x \leq n}\left|p^{\prime}(x)\right|$, which is greater than or equal to $c$. It is also clear that for all real $0 \leq x \leq n$,

$$
b_{1}-\frac{c^{\prime}}{2} \leq p(x) \leq b_{2}+\frac{c^{\prime}}{2} .
$$

Using the Markov inequality, we have

$$
\begin{gathered}
c^{\prime} \leq \frac{\operatorname{deg}(p)^{2}\left(c^{\prime}+b_{2}-b_{1}\right)}{n} \\
\Longrightarrow \operatorname{deg}(p)^{2} \geq \frac{c^{\prime} n}{c^{\prime}+b_{2}-b_{1}} \geq \frac{c n}{c+b_{2}-b_{1}} .
\end{gathered}
$$


Lemma 4.21. Let $f$ be a Boolean function such that $f(000 \cdots 0)=0$ and for every Boolean vector $\mathbf{x}$ of Hamming weight $1, f(\mathbf{x})=1$. Then

$$
\operatorname{deg}(f) \geq \sqrt{\frac{n}{2}}
$$

and

$$
\widetilde{\operatorname{deg}}(f) \geq \sqrt{\frac{n}{6}}
$$

Proof. We will prove the bound for $\widetilde{\operatorname{deg}}(f)$. Let $p$ be a univariate polynomial approximating $f$, and consider $\tilde{p}$ the univariate polynomial giving its symmetrization. $\tilde{p}$ satisfies the following properties.

(1) By Lemma $4.18 \operatorname{deg}(\tilde{p}) \leq \operatorname{deg}(p)$.

(2) For every Boolean vector $\mathbf{x}$, the function $p(\mathbf{x})$ is within $\frac{1}{3}$ of a Boolean value (by equation (77)), so for every integer $0 \leq i \leq n,-\frac{1}{3} \leq \tilde{p}(i) \leq \frac{4}{3}$.

(3) Since, $f(000 \cdots 0)=0$, we get $\tilde{p}(0) \leq \frac{1}{3}$ by equation (7).

(4) Since for all Boolean vectors $\mathbf{x}$ of Hamming weight $1, f(\mathbf{x})=1$. Using equation (7) once again, we get $\tilde{p}(1) \geq \frac{2}{3}$.

By properties (3) and (4) above and by using the mean value theorem (MVT) for derivatives, we obtain for some real $0 \leq z \leq 1$, the derivative $\tilde{p}^{\prime}(z) \geq \frac{1}{3}$. We can apply Lemma 4.20 to obtain the lower bound for $\operatorname{deg}(\tilde{p})$. We can also get a similar bound for $\operatorname{deg}(p)$ by using $0 \leq \tilde{p}(i) \leq 1$ along with Lemma 4.20 and the MVT for derivatives.

Example 4.22. There exists an (explicitly given) Boolean function $f$ of $n$ variables satisfying $f(\mathbf{0})=0$ and $f\left(e_{i}\right)=1$ and $\operatorname{deg}(f)=n^{\alpha}$ for $\alpha=\log _{3} 2=0.631 \cdots$.

We can show this by considering a function on three variables that is true if exactly one or two of the input bits are true. Then, we define a Boolean function $f_{m}$ on $n=3^{m}$ variables obtained by building a complete ternary tree of depth $m$. Analyzing $\operatorname{deg}\left(f_{m}\right)$ gives the required result.

Nisan [6] had previously established a polynomial relation between the block sensitivity and the decision-tree complexity as

$$
b s(f) \leq D(f) \leq b s^{4}(f) .
$$

Lemma 4.23. For every Boolean function $f$,

$$
\operatorname{deg}(f) \geq \sqrt{\frac{b s(f)}{2}}
$$

and

$$
\widetilde{\operatorname{deg}}(f) \geq \sqrt{\frac{b s(f)}{6}} .
$$

Proof. We refer to [28, Theorem 14.11] for the proof of this lemma.

Let $f(x)$ be a Boolean function on $n$ variables, and let $q: \mathbb{R}^{n} \rightarrow \mathbb{R}$ be the multilinear polynomial of degree $d$ representing $f$. By Lemma 4.21, we know that every Boolean function $f$ of $n$ variables, which rejects the zero vector and accepts all $n$ vectors with Hamming weight 1 , has $\operatorname{deg}(f) \geq \sqrt{\frac{n}{2}}$. It is therefore enough to construct a multilinear polynomial $p$ of $t=b s(f)$ variables satisfying the conditions of Lemma 4.21 to prove (19). 
Let $t=b s(f)$, and let $a$ and $S_{1}, \ldots, S_{t}$ be the input and the sets achieving the block sensitivity. Assume without loss of generality that $f(a)=0$. We transform $q\left(x_{1}, x_{2}, \ldots, x_{n}\right)$ into a multilinear polynomial $p\left(y_{1}, y_{2}, \ldots, y_{t}\right)$ of $t$ new variables by replacing every variable $x_{j}$ in $p$ as follows. Define a function $f^{\prime}\left(y_{1}, \ldots, y_{t}\right)$ as

$$
x_{j}:= \begin{cases}y_{i}, & \text { if } a_{j}=0 \text { and } j \in S_{i} \\ 1-y_{i}, & \text { if } a_{j}=1 \text { and } j \in S_{i} \\ a_{j} & \text { if } j \notin S_{1} \cup \cdots \cup S_{t} .\end{cases}
$$

That is, for $y \in\{0,1\}^{t}$ we have that

$$
p(y)=q\left(a \oplus y_{1} S_{1} \oplus \cdots \oplus y_{t} S_{t}\right),
$$

where

$$
y_{i} S_{i}=(0, \ldots, 0, \overbrace{y_{1}, \ldots, y_{i}}^{S_{i}}, 0, \ldots, 0) .
$$

We can easily verify that $p$ is a multilinear polynomial of degree at most $d$ and satisfies the conditions of Lemma 4.21. We can therefore conclude that

$$
d=\operatorname{deg}(q) \geq \operatorname{deg}(p) \geq \sqrt{\frac{t}{2}}=\sqrt{\frac{b s(f)}{2}}
$$

The proof of the other part is analogous.

Since $\operatorname{deg}(f) \leq D(f)$ is obvious (note that the tests along paths to 1-leaves define a multilinear polynomial), by combining (18) and the results of Lemma 4.23, we have (9) and (10).

\section{Gotsman and Linial's Result}

In this section, we look at Gotsman and Linial's result 1 that reduces the sensitivity conjecture to answering a "simple" question about cubes of different dimensions: If you choose any collection of more than half the corners of a cube and color them red, is there always some red point that is connected to many other red points?

5.1. Reformulating the sensitivity conjecture. Consider a Boolean function $f:\{-1,1\}^{n} \rightarrow\{-1,1\}$. Let $g$ be the same function as $f$ except that we flip the value on all odd vertices. Notice that the sensitivity of $f$ on $x$ is the number of $i$ such that

$$
g\left(x_{1}, \ldots, x_{i}, \ldots, x_{n}\right)=g\left(x_{1}, \ldots,-x_{i}, \ldots, x_{n}\right) .
$$

This can be easily visualized as follows. Take a Boolean function $f$ and for any $x \in Q_{n}$, calculate $f(x)$, marking the vertex blue if $f(x)=1$ and red, otherwise. Thus, the sensitivity of $f$ on $x$ is the number of neighbors of $x$ in $Q_{n}$ that have a different color than $x$.

Take $G$ to be the induced subgraph on the set of $x$ such that $g(x)=-1$ if the node $x$ was colored blue and $H$ to be the induced subgraph on the set of $x$ such that $g(x)=1$ if the node $x$ was colored red. The sensitivity of $f$, using this new notation, is nothing but the maximum number of neighbors of any vertex in $G$ or $H$. Recall the representation of $f$ as a multilinear polynomial over the reals that we saw in section 4. The sensitivity conjecture states that there is some $\alpha>0$ such that if $f$ has degree $n$, then $f$ has sensitivity at least $n^{\alpha}$. 
Let us denote $\Gamma(G)=\max \left(\Delta(G), \Delta\left(Q_{n} \backslash G\right)\right)$. Gotsman and Linial proved the following equivalence that helps in forming an upper bound for $d(f)$ in terms of $s(f)$.

Theorem 5.1. The following are equivalent for any function $h: \mathbb{N} \rightarrow \mathbb{R}$.

1. For any induced subgraph $G$ of $Q_{n}$ such that $|V(G)| \neq 2^{n-1}, \Gamma(G) \geq h(n)$.

2. For any Boolean function $f, h(d(f))<s(f)$.

Remark 5.2. Szegedy [14] proved a tight lower bound for $d(f)$ in terms of $s(f)$ as

$$
d(f) \geq \sqrt{s(f)} .
$$

Remark 5.3. In graph-theoretical terms: Suppose you have a partition of the hypercube graph $Q_{n}$ into sets $A$ and $B$ such that $|A| \neq|B|$, and let $G$ and $H$ be the induced subgraphs of $A$ and $B$. Then, there is some constant $\alpha>0$ such that there is a node of $A$ or $B$ with at least $n^{\alpha}$ neighbors.

Remark 5.4. Hao proved that given any subset $A$ of the vertices of a hypercube with $|A|>2^{n-1}$, the induced subgraph has a node of degree at least $\sqrt{n}$. Since either $A$ or $B$ in the Gotsman-Linial assumption has size greater than $2^{n-1}$, Hao's result proves the sensitivity conjecture.

Proof. Let us convert statement 1 of Theorem 5.1 into a statement on Boolean functions. Associate with the induced subgraph $G$ a Boolean function $g$ such that $g(x)=1$ iff $x \in V(G)$. One way to do this would be to use Karnaugh maps. Observe that $\operatorname{deg}_{G}(x)=n-s(g, x)$ for $x \in V(G)$, and the same relation also holds in $Q_{n} \backslash G$ for $x \notin V(G)$.

Denote by $\mathbf{E}(g)=2^{-n} \sum_{x} g(x)$ the average value of $g$ on $Q_{n}$. Now, statements 1 and 2 of Theorem 5.1 are equivalent to:

Lemma 5.5. Theorem 5.1 can be reformulated as

I. For any Boolean function $g, \mathbf{E}(g) \neq 0 \Longrightarrow \exists x: s(g, x) \leq n-h(n)$.

II. For any Boolean function $f, s(f)<h(n) \Longrightarrow d(f)<n$.

Proof. Proving $1 \rightarrow \mathrm{I}$.

$$
\begin{aligned}
\Gamma(G)=\max \left(\Delta(G), \Delta\left(Q_{n} \backslash G\right)\right) \geq h(n) & \Longrightarrow \Delta(G) \geq h(n) \\
& \Longrightarrow \max _{x \in V(G)} \operatorname{deg}_{G}(x) \geq h(n) \\
& \Longrightarrow \exists x: \operatorname{deg}_{G}(x) \geq h(n) \\
& \Longrightarrow \exists x: n-s(g, x) \geq h(n) \\
& \Longrightarrow \exists x: s(g, x) \leq n-h(n),
\end{aligned}
$$

where the first implication is done assuming that $\Delta(G) \geq \Delta\left(Q_{n} \backslash G\right)$.

Proving $\mathrm{I} \rightarrow 1$. The requirement of $\mathbf{E}(g) \neq 0$ corresponds to $|V(G)| \neq 2^{n-1}$. Reversing the implications of the previous result prove the necessary result.

Proving $2 \rightarrow$ II. Given $d(f)<h^{-1}(s(f))$, the result follows naturally if $s(f)<h(n)$. $\overline{\text { Proving II } \rightarrow 2}$. To prove the reverse implication, let $f$ be a Boolean function of

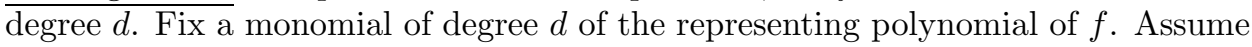
without loss of generality that the monomial is $x_{1} \cdots x_{d}$. Define $g\left(x_{1}, \ldots, x_{d}\right):=$ $f\left(x_{1}, \ldots, x_{d}, 0, \ldots, 0\right)$. Then, $g$ has full degree $d$, so it follows that $s(f) \geq s(g) \geq$ $h(d)=h(\operatorname{deg} f)$, as desired. 
To see the equivalence of I and II, define

$$
g(x)=f(x) p(x),
$$

where $p(x)=(-1)^{\sum x_{i}}$ is the parity function (note that we take the range of Boolean functions to be $\{-1,+1\})$. Since the parity function is sensitive to all $n$ variables, observe that $\forall x \in Q_{n}$,

$$
s(g, x)=n-s(f, x),
$$

and for all $S \subseteq[n]$,

$$
\hat{g}(S)=2^{-n} \sum_{x} g(x) \prod_{i \in S} x_{i}=2^{-n} \sum_{x} f(x) \prod_{i \notin S} x_{i}=\hat{f}([n] \backslash S) .
$$

In particular,

$$
\mathbf{E}(g)=\hat{g}(\phi)=\hat{f}([n]),
$$

where $\hat{f}(S)$ is the highest order Fourier coefficient in the polynomial representation of $f$.

Proving I $\rightarrow$ II. Assume that $d(f)=n$, i.e., $\hat{f}([n]) \neq 0$. By (23), $\mathbf{E}(g) \neq 0$ and by $\mathrm{I}$,

$$
\exists x: s(g, x) \leq n-h(n) \Longrightarrow \exists x: s(f, x) \geq h(n),
$$

a contradiction.

Proving II $\rightarrow$ I. Assume

$$
\forall x: s(g, x)=n-s(f, x)>n-h(n) \Longrightarrow s(f)<h(n)
$$

as $s(f)=\max _{x \in Q_{n}} s(f, x)$. Then by II,

$$
d(f)<n \Longrightarrow \hat{f}([n])=\hat{g}(\phi)=\mathbf{E}(g)=0,
$$

a contradiction.

5.2. Consequences. Gotsman and Linial's result translates a Boolean function with a polynomial gap between degree and sensitivity into a graph with the same polynomial gap between $\Gamma$ and $n$, and vice versa. For example, observe that Rubinstein's function (section 5.2.1) can be used to obtain a graph $G$ with the surprising property $\Gamma(G)=\Theta(\sqrt{n})$. Also, we constructed a graph $G$ with $\Gamma(G)<\sqrt{n}+1$ in section 3.1. Theorem 5.1 implies that the following conjecture is equivalent to the sensitivity conjecture.

Conjecture 5.6. There is a constant $\epsilon>0$ such that for every induced subgraph $G$ of $Q_{n}$ with $|V(G)| \neq 2^{n-1}$, we have $\Gamma(G) \geq n^{\epsilon}$.

5.2.1. Rubinstein's function. The following function was constructed by Rubinstein [11]: Take $n=k^{2}$ variables ( $k$ even), divided into $k$ blocks of $k$ variables each. The value of the function is 1 if there is at least one block with exactly two consecutive 1 's in it, and 0 otherwise.

The block sensitivity of Rubinstein's function is equal to $n / 2$ (hence, the certificate complexity and the decision-tree complexity is at least $n / 2$ ) and the sensitivity is $\sqrt{n}$; this can be verified by a direct computation of $\hat{f}([n])$. Hence, one can establish for this function that

$$
b s(f) \geq s(f)^{2} / 2 .
$$




\section{HAO'S RESULT}

In this section, we expound Hao's result [4] which shows that every $\left(2^{n-1}+1\right)$ vertex induced subgraph of the $n$-cube graph $Q_{n}$ has maximum degree at least $\sqrt{n}$, hence proving the sensitivity conjecture.

Rubinstein's example (see section 5.2.1) is still the best-known lower bound on $b s(f)$ in terms of $s(f)$. But no one had proven anything better than an exponential upper bound until Hao's result, from which it follows that: For all Boolean functions $f$

$$
b s(f) \leq 2 s(f)^{4} .
$$

Observe that this concrete bound is the combination of two quadratic bounds. From (19), we conclude that

$$
b s(f) \leq 2 \operatorname{deg}(f)^{2} .
$$

The other is the conjecture (take $h(x)=\sqrt{x}$ in Theorem [5.1),

$$
\operatorname{deg}(f) \leq s(f)^{2},
$$

which is what Hao proves (Theorem 1.4).

\subsection{Cauchy's interlace theorem.}

Lemma 6.1. All eigenvalues of a Hermitian matrix are real numbers.

Proof. Let $\lambda$ be an arbitrary eigenvalue of a Hermitian matrix $A$ with corresponding eigenvector $\mathbf{x}$. Then

$$
A \mathbf{x}=\lambda \mathbf{x} \Longrightarrow \mathbf{x}^{T} A \mathbf{x}=\mathbf{x}^{T}(\lambda \mathbf{x}) \Longrightarrow \mathbf{x}^{T} A \mathbf{x}=\lambda \mathbf{x}^{T} \mathbf{x}=\lambda\|\mathbf{x}\| .
$$

Take the conjugate transpose on both sides to get (note $A$ is Hermitian)

$$
\mathbf{x}^{T} \bar{A}^{T} \mathbf{x}=\bar{\lambda}\|\mathbf{x}\| \Longrightarrow \bar{\lambda}\|\mathbf{x}\|=\mathbf{x}^{T} \bar{A}^{T} \mathbf{x}=\mathbf{x}^{T} A \mathbf{x}=\mathbf{x}^{T} \lambda \mathbf{x}=\lambda\|\mathbf{x}\| .
$$

Hence, $\lambda\|\mathbf{x}\|=\bar{\lambda}\|\mathbf{x}\|$. Being an eigenvector, $\mathbf{x}$ cannot be the zero vector. So, $\bar{\lambda}=\lambda \Longrightarrow \lambda \in \mathbb{R}$.

If $f$ and $g$ are polynomials with real roots $r_{1} \leq r_{2} \leq \cdots \leq r_{n}$ and $s_{1} \leq s_{2} \leq \cdots \leq$ $s_{n-1}$, we say that $f$ and $g$ interlace if and only if $r_{1} \leq s_{1} \leq r_{2} \leq \cdots \leq s_{n-1} \leq r_{n}$.

Lemma 6.2 ([29, chapter 6$])$. The roots of polynomials $f$ and $g$ interlace if and only if the linear combinations $f+\alpha g$ have all real roots for all $\alpha \in \mathbb{R}$.

Corollary 6.3. If $A$ is a Hermitian matrix and $B$ is a principal submatrix of $A$, then the eigenvalues of $B$ interlace the eigenvalues of $A$.

Proof. We will need another definition.

Definition 6.4. Given a real $n \times n$ matrix $A$, a principal submatrix of $A$ is obtained by deleting the same set of rows and columns of $A$.

By simultaneously permuting rows and columns, if necessary, we may assume that the submatrix $B$ occupies rows $2, \ldots, n$ and columns $2, \ldots, n$, so that $A$ has the form

$$
A=\left(\begin{array}{ll}
B & \mathbf{c} \\
\mathbf{c}^{*} & d
\end{array}\right)
$$


where $*$ signifies the conjugate transpose of a matrix. Choose $\alpha \in \mathbf{R}$, and consider the equation that follows from the linearity of the determinant,

$$
\left|\begin{array}{cc}
B-x I & \mathbf{c} \\
\mathbf{c}^{*} & d-x+\alpha
\end{array}\right|=\left|\begin{array}{cc}
B-x I & \mathbf{c} \\
\mathbf{c}^{*} & d-x
\end{array}\right|+\left|\begin{array}{cc}
B-x I & \mathbf{c} \\
0 & \alpha
\end{array}\right| .
$$

Using Lemma 6.1 on the right-hand side of (26) implies that $|A-x I|+\alpha|B-x I|$ has all real roots for any $\alpha$, so the eigenvalues interlace.

Consequently,

Theorem 6.5 (Cauchy's Interlace Theorem, Fisk [12]). Let $A$ be a symmetric $n \times n$ matrix, and let $B$ be a $m \times m$ submatrix of $A$ for some $m<n$. If the eigenvalues of $A$ are $\lambda_{1} \geq \lambda_{2} \geq \cdots \geq \lambda_{n}$ and the eigenvalues of $B$ are $\mu_{1} \geq \mu_{2} \geq \cdots \geq \mu_{m}$, for all $1 \leq i \leq m$,

$$
\lambda_{i} \geq \mu_{i} \geq \lambda_{i+n-m}
$$

6.2. Proving the sensitivity conjecture. Our discussion of Hao's proof of the conjecture is inspired by [30. One can show that the degree $d(G)$ of a graph $G$ is always at least as great as the largest eigenvalue $\lambda_{1}$ of the adjacency matrix $A_{G}$. Take an $m$-vertex subgraph $H$ of $Q_{n}$, and let its top eigenvalue be $\lambda_{1}(H)$. We know that the entries of the adjacency matrix $A_{H}$ are either 0 or 1 . Hao's insight was to realize that if one flipped the signs of some 1 's in $A_{H}$, thus creating a so-called pseudo-adjacency matrix, the relation between the degree and the largest eigenvalue of the adjacency matrix still holds.

Lemma 6.6. Suppose $H$ is an m-vertex undirected graph and $A$ is a symmetric matrix with entries in $\{0, \pm 1\}$ and whose rows and columns are indexed by $V(H)$, the vertex set of $H$. Also, let $A_{u, v}=0$ whenever $u, v \in V(H)$ are nonadjacent. Then,

$$
\Delta(H) \geq \lambda_{1}:=\lambda_{1}(A) .
$$

Proof. Let $\mathbf{v}$ be the eigenvector corresponding to $\lambda_{1}$. Then, $\lambda_{1} \mathbf{v}=A \mathbf{v}$. Choose an index $i$ that maximizes the absolute value $\left|v_{i}\right|$. Then

$$
\left|\lambda_{1} v_{i}\right|=\left|(A \mathbf{v})_{i}\right|=\left|\sum_{j} A_{i, j} v_{j}\right| \leq\left|\sum_{j} A_{i, j}\right| \cdot\left|v_{i}\right| \leq \sum_{(i, j) \in E(G)}\left|A_{i, j}\right| \cdot\left|v_{i}\right| \leq d(H)\left|v_{i}\right|
$$

from which the result follows.

Observe that a key idea in Lemma 6.6 was the introduction of -1 entries in the adjacency matrix $A_{H}$. What would happen if we try to use the original (unmodified) adjacency matrix in Lemma 6.6. One readily observes that although this matrix has its top eigenvalue at least $\sqrt{n}$, the interlacing bound is too lossy to prove this without modification. Take for example, the three-dimensional cube $Q_{3}$, in which case, the eigenvalues of the unmodified adjacency matrix are $\{-3,-1,-1,-1,1,1,1,3\}$, giving the trivial bound $\lambda_{1} \geq 0$. In general, the eigenvalues of the adjacency matrix of $Q_{n}$ will be the integers $-n,-n+2,-n+4, \ldots, n$.

We now want to find conditions that force $\lambda_{1}=\sqrt{n}$, particularly when $m \geq \frac{N}{2}+1$ with $N=\left|V\left(Q_{n}\right)\right|=2^{n}$. Hao does this by making $A$ sit inside a matrix $A_{N}$ (whose construction is discussed in section 6.3) with at least $\frac{N}{2}$ eigenvalues with value $\sqrt{n}$.

For realizing this, construct $A_{N-1}$ as a principal submatrix of $A_{N}$ by deleting its last row and column. Since $A_{N}$ and $A_{N-1}$ are both real and symmetric matrices, they have real eigenvalues, and so we can order them as $\lambda_{1}, \ldots, \lambda_{N}$ and $\mu_{1}, \ldots, \mu_{N-1}$ 
in nonincreasing order. Note that their eigenvalues always interlace following from the property outlined before Lemma 6.2.

Observe that this process can be repeated; that is, we can construct $A_{N-2}$ by knocking off another row and its corresponding column. Denoting the eigenvalues of $A_{N-2}$ by $\nu_{i}$, we can easily see that

$$
\mu_{1} \geq \nu_{1} \geq \mu_{2} \geq \nu_{2} \geq \mu_{3} \cdots \Longrightarrow \lambda_{1} \geq \nu_{1} \geq \lambda_{3} .
$$

Repeating this procedure gives us another matrix whose top eigenvalue is still at least as big as $\lambda_{4}$.

Generalizing this, if we repeat this process $\frac{N}{2}-1=2^{n-1}-1$ times inside $A_{N}$, the resulting matrix's largest eigenvalue is still at least as large as $\lambda_{\frac{N}{2}}\left(A_{N}\right)$, which we assign the value of $\sqrt{n}$. This means the size of the resultant (square) matrix will be $m=\frac{N}{2}+1=2^{n-1}+1$, and consequently

$$
\lambda_{1}\left(A_{N}\right) \geq \lambda_{1}\left(A_{m}\right) \geq \lambda_{\frac{N}{2}}\left(A_{N}\right)=\sqrt{n},
$$

which when used with Lemma 6.6 yields

$$
d(H) \geq \sqrt{n}
$$

thus proving Theorem 1.4

6.3. Constructing the matrix. As noted earlier, Hao showed that there exists a $N \times N$ matrix $A_{N}$ with entries in $\{0, \pm 1\}$ whose nonzero entries correspond to the edges of the Boolean cube and such that all the $N$ eigenvalues of $A_{N}$ are $\pm \sqrt{n}$. As a result, these eigenvalues sum up to zero, meaning that the trace is zero. This would then imply that $A_{N}^{2}=n I$.

We would have ideally wanted $A_{N}$ to be the adjacency matrix of the $n$-cube but that does not work-because each $(i, j)$ entry of the square of the unmodified induced adjacency matrix of $Q_{n}$ counts all paths of length 2 from node $i$ to node $j$, and that number is nonzero.

This is where the introduction of -1 on edges comes in handy. We arrange that every 4 -cycle of the $n$-cube has exactly one edge with -1 . Then, the pairs of paths from one corner to the opposite corner will always cancel, leaving $A_{i, j}^{2}=0$ whenever $i \neq j$. And $A_{i, i}^{2}=n$ because there are $n$ ways to go out and come back along the same edge, always contributing $1 \cdot 1$ or $(-1) \cdot(-1)$ either way.

Subsequently, Hao defines the needed labeling exactly by the recursion,

$$
A_{2}=\left[\begin{array}{ll}
0 & 1 \\
1 & 0
\end{array}\right] \text { and } A_{N}=\left[\begin{array}{cc}
A_{\frac{N}{2}} & I \\
I & -A_{\frac{N}{2}}
\end{array}\right] \text { for }(N>2) .
$$

One can easily verify that $A_{N}^{2}=n I$ by induction. A nice exposition of the physical interpretation of this pseudo-adjacency matrix, using physics concepts such as the Jordan-Wigner transformation and Majorana fermions, is given in [13].

\section{FURTHER DISCUSSION}

In this section we provide comments on the open problems posed by Hao [4].

Definition 7.1. An automorphism of $G$ is a bijection $f: V(G) \rightarrow V(G)$ sending edges to edges and nonedges to nonedges. 
Denote by $H=$ Aut $G$ the full automorphism group of $G$. Roughly speaking, the more symmetry a graph has, the larger its automorphism group will be, and vice versa.

Definition 7.2. The graph $G$ is vertex-transitive if $H$ acts transitively on $V(G)$, that is, for all $u, v \in V(G)$, there is an automorphism $f \in H$ such that $f(u)=v$.

Intuitively, a graph is vertex-transitive if there is no structural (i.e., nonlabeling) way to distinguish vertices of the graph. Some examples include the complete graph $K_{n}$ and the cycle $C_{n}$ on $n$ vertices. For a not-so-obvious example, let $G$ be a group, and let $S \subset G$ be a generating set for $G$ such that $1_{G} \notin S$ and $S$ is closed under taking inverses.

Definition 7.3. The (right) Cayley graph $\Gamma=\Gamma(G, S)$ is given by

$$
V(\Gamma)=G ; \quad E(\Gamma)=\left\{\{g, h\}: g^{-1} h \in S\right\} .
$$

Thus, two vertices are adjacent if they differ in $G$ by right multiplication by a generator. The Cayley graph of a group is always vertex-transitive.

\subsection{Three notions of symmetry.}

7.1.1. Distance-transitive graphs. In a connected graph $G$, define the distance $d(u, v)$ between $u, v \in V(G)$ as the length of the shortest path from $u$ to $v$.

Definition 7.4 (Gray [15]). A graph is distance-transitive if for any two pairs of vertices $(u, v)$ and $\left(u^{\prime}, v^{\prime}\right)$ with $d(u, v)=d\left(u^{\prime}, v^{\prime}\right)$, there is an automorphism taking $u$ to $u^{\prime}$ and $v$ to $v^{\prime}$.

It is seen that distance-transitivity implies vertex-transitivity. The Hamming graph $H(n, k)$ is defined by the vertex set

$$
\mathbf{Z}_{k}^{n}=\underbrace{\mathbf{Z}_{k} \times \cdots \times \mathbf{Z}_{k}}_{n \text { times }}
$$

where $\mathbf{Z}_{k}=[k-1]=\{0,1, \ldots, k-2, k-1\}$ and two vertices $u$ and $v$ are adjacent iff they differ in exactly one coordinate. The $d$-dimensional hypercube is defined to be $H(n, 2):=Q_{d}$. It is observed that Hamming graphs are a family of distancetransitive graphs (see [16, Chapter 5]).

A connected, acyclic graph is called a tree. A regular tree $T_{r}(r \in \mathbb{N})$, where all vertices have degree $r$, is an example of an infinite locally finite (all vertices having finite degree) distance-transitive graph. Macpherson's theorem [17] gives a necessary and sufficient condition for a locally finite infinite graph to be distancetransitive.

\subsubsection{Homogeneous graphs.}

Definition 7.5 (15). A graph $G$ is called homogeneous if any isomorphism between finite induced subgraphs extends to an automorphism of the graph.

Homogeneity is the strongest possible symmetry condition we can impose. Gardiner's result [18] gives a concrete classification of finite homogeneous graphs, and for the infinite case we cite an example of a random graph $R$ constructed by Rado [19]. 


\subsubsection{Connected-homogeneous graphs.}

Definition 7.6 ([15]). A graph $G$ is connected-homogeneous if any isomorphism between connected finite induced subgraphs extends to an automorphism.

This helps us define an intermediate class of graphs between the homogeneous and distance-transitive graphs. Gardiner [20] gives yet another concrete classification of finite connected-homogeneous graphs. A result of Gray and Macpherson 21] helps us to classify the countable connected-homogeneous graphs.

\subsection{Problems.}

Problem 7.7. Given a graph $G$ with high symmetry, what can we say about the smallest maximum degree of induced subgraphs of $G$ with $\alpha(G)+1$ vertices, where $\alpha(G)$ denotes the size of the largest independent set in $G$ ?

Definition 7.8. A set of vertices in $G$ is an independent set if no two vertices in the set are adjacent.

Definition 7.9. A maximal independent set is an independent set to which no other vertex can be added without destroying its independence property. The number of vertices in the largest independent set of $G$ is called the independence number, $\alpha(G)$.

Chung et al.'s result (see section 3), that the smallest maximum degree of $Q_{n}$ is at most $\lceil\sqrt{n}\rceil$, has been generalized in 22 for Hamming graphs $H(n, k)$ for all $n, k \geq 1$. It has been observed that the same result holds true for these graphs also, giving a bound independent of the value of $k$.

It would be interesting to find whether the classes of symmetric graphs described above yield analogous results.

Problem 7.10. Let $g(n, k)$ be the minimum $t$ such that every $t$-vertex induced subgraph $H$ of $Q_{n}$ has maximum degree at least $k$. Hao showed that $g(n, \sqrt{n})=$ $2^{n-1}+1$. Can we determine $g(n, k)$ (asymptotically) for other values of $k$ ?

We are unaware of any prior work that has considered the quantity $g(n, k)$. However, Agnarsson [23] analyses the maximum number of vertices of degree $k$ in an induced subgraph on $n$ vertices of $Q_{k}$.

Problem 7.11. The best separation between the block sensitivity $b s(f)$ and the sensitivity $s(f)$ is $b s(f)=\frac{2}{3} s(f)^{2}-\frac{1}{3} s(f)$, which is quadratic (see [24]). Hao proves a quartic upper bound. Is it possible to close this gap by directly applying the spectral method to Boolean functions instead of to the hypercubes?

One might try to see what happens if we tweak $b s(f)=O(s(f))^{4}$, which was the upper bound proved by Hao, to say, $O(s(f))^{2}$. To check its validity, we first look at the two results used by Hao to show $b s(f)=O(s(f))^{4}$. The first one, shown by Hao, which essentially proved Gotsman and Linial's conjecture (see (25)) was that for all Boolean functions $f:\{0,1\}^{n} \rightarrow\{0,1\}$, the inequality $s(f) \geq \sqrt{\operatorname{deg}(f)}$ holds. This is seen to be a tight bound for the AND-of-ORs function:

Definition 7.12. The AND-of-ORs function is defined on $n$ blocks of $n$ variables each as

$$
f\left(x_{11}, \ldots, x_{n n}\right)=\bigwedge_{i=1}^{n} \bigvee_{i=1}^{n} x_{i j}
$$


This inequality is combined with Nisan and Szegedy's result (see (19)) stating that for all $f:\{0,1\}^{n} \rightarrow\{0,1\}, \operatorname{deg}(f) \geq \sqrt{\frac{1}{2} b s(f)}$. From Hatami's survey [10], it is known that this bound cannot be improved beyond $b s(f) \gtrsim(\operatorname{deg} f)^{\log _{3} 6}$, where $\log _{3} 6 \approx 1.6309$. So, the use of $\operatorname{deg}(f)$ is not feasible to try to get a quadratic sensitivity upper bound on the block sensitivity.

It is still open as to whether exploring the techniques of interlacing eigenvalues of signed matrices on different objects may lead to sharper bounds.

Note 7.13. It would be instructive to mention the connection of Hao's proof to Clifford algebras as shown in 25]. This concept has been used to extend Hao's result for a Cartesian power of a directed $l$-cycle in [26].

\section{SEnsitivity AND CREW PRAMs}

In this section we discuss how the sensitivity conjecture is related to CREW PRAMs.

A PRAM (parallel random access machine) is the standard model for parallel computation. It consists of a set of processors $P_{0}, P_{1}, \ldots$ which communicate by means of cells $C_{0}, C_{1}, \ldots$ of shared memory. Each step of computation (of a function $f$ ) consists of three phases: read, compute, and write phases. In the read phase, each processor may choose one cell to read from. In the compute phase, an arbitrary amount of local computation can take place. In the write phase, each processor may choose one cell to write into.

In the CREW (concurrent read exclusive write) PRAM variant, simultaneous read access is permitted, but not simultaneous write access. That is, several processors may read from the same location at the same time, but two or more processors may never attempt writing into the same location at the same time.

A key result bounding the power of ideal CREW PRAMs is by Cook, Dwork, and Reischuk [8] where they show that CREW $(f)=\Omega(\log (s(f)))$ is a lower bound on the number of steps required to compute a function $f$ on a CREW PRAM. After this, Nisan [6] tweaked the definition of sensitivity and introduced the notion of block sensitivity to obtain $\mathrm{CREW}(f)=\Theta(\log (b s(f)))$. Now, one would ask naturally whether CREW $(f)=O(\log (s(f)))$ ? Proving this is the sensitivity conjecture.

\section{ACKNOWLEDGMENTS}

We would like to thank our project advisor, Dr. Chandra Prakash Singh for his constant encouragement in the preparation of this manuscript. We thank the anonymous referee for providing useful suggestions on earlier versions of this paper. We would also like to thank Quanta Magazine [31] for introducing us to this wonderful topic of sensitivity. Last but not the least, we would like to thank our parents for providing constant moral support.

\section{ABout THE AUTHORS}

Rohan Karthikeyan is an undergraduate student at Delhi Techonological University. His research interests lie in exploring interconnections between topics in algebra and combinatorics.

Siddharth Sinha is an undergraduate student at Delhi Techonological University. His research interests lie in in algebra, specifically group theory. 
Vallabh Patil is an undergraduate student at Delhi Techonological University. His research interests include machine learning, algorithms, and data structures.

\section{REFERENCES}

[1] C. Gotsman and N. Linial, The equivalence of two problems on the cube, J. Combin. Theory Ser. A 61 (1992), no. 1, 142-146, DOI 10.1016/0097-3165(92)90060-8. MR1178392

[2] F. R. K. Chung, Z. Füredi, R. L. Graham, and P. Seymour, On induced subgraphs of the cube, J. Combin. Theory Ser. A 49 (1988), no. 1, 180-187, DOI 10.1016/0097-3165(88)900349. MR957216

[3] N. Nisan and M. Szegedy, On the degree of Boolean functions as real polynomials, Comput. Complexity 4 (1994), no. 4, 301-313, DOI 10.1007/BF01263419. MR.1313531

[4] H. Huang, Induced subgraphs of hypercubes and a proof of the sensitivity conjecture, Ann. of Math. (2) 190 (2019), no. 3, 949-955, DOI 10.4007/annals.2019.190.3.6. MR4024566

[5] R. O'Donnell, Some topics in analysis of Boolean functions, STOC'08, ACM, New York, 2008, pp. 569-578, DOI 10.1145/1374376.1374458. Tutorial. MR.2582688

[6] N. Nisan, CREW PRAMs and decision trees, SIAM J. Comput. 20 (1991), no. 6, 999-1007, DOI 10.1137/0220062. MR1135744

[7] J. Kahn, G. Kalai, N. Linial. The influence of variables on boolean functions. FOCS (1988), 68-80.

[8] S. Cook, C. Dwork, and R. Reischuk, Upper and lower time bounds for parallel random access machines without simultaneous writes, SIAM J. Comput. 15 (1986), no. 1, 87-97, DOI 10.1137/0215006. MR822194

[9] H. Buhrman and R. de Wolf, Complexity measures and decision tree complexity: a survey, Theoret. Comput. Sci. 288 (2002), no. 1, 21-43, DOI 10.1016/S0304-3975(01)00144-X. Complexity and logic (Vienna, 1998). MR 1934888

[10] P. Hatami, R. Kulkarni, D. Pankratov. Variations on the Sensitivity Conjecture. Theory of Computing Library, Graduate Surveys 4 (2011), 1-27.

[11] D. Rubinstein, Sensitivity vs. block sensitivity of Boolean functions, Combinatorica 15 (1995), no. 2, 297-299, DOI 10.1007/BF01200762. MR1337360

[12] S. Fisk. A very short proof of Cauchy's interlace theorem for eigenvalues of Hermitian matrices. Amer. Math. Monthly, 112 (2) (2005), 118.

[13] Y. Gu, X.-L. Qi. Majorana fermions and the Sensitivity Conjecture, arXiv:1908.06322 (2019).

[14] M. Szegedy. Algebraic methods in Lower Bounds for Computational Models with Limited Communication. Ph. D. thesis, University of Chicago, 1989.

[15] R. Gray. Graphs with a high degree of symmetry (PowerPoint slides). Available at: https:// archive.uea.ac.uk/ fga12juu. (2007)

[16] R. A. Bailey. Distance-transitive graphs. M. Math dissertation, University of Leeds, 2002.

[17] H. D. Macpherson, Infinite distance transitive graphs of finite valency, Combinatorica 2 (1982), no. 1, 63-69, DOI 10.1007/BF02579282. MR671146

[18] A. Gardiner, Homogeneous graphs, J. Combinatorial Theory Ser. B 20 (1976), no. 1, 94-102, DOI 10.1016/0095-8956(76)90072-1. MR419293

[19] R. Rado, Universal graphs and universal functions, Acta Arith. 9 (1964), 331-340, DOI 10.4064/aa-9-4-331-340. MR.172268

[20] A. Gardiner, Homogeneity conditions in graphs, J. Combin. Theory Ser. B 24 (1978), no. 3, 301-310, DOI 10.1016/0095-8956(78)90048-5. MR496449

[21] R. Gray and D. Macpherson, Countable connected-homogeneous graphs, J. Combin. Theory Ser. B 100 (2010), no. 2, 97-118, DOI 10.1016/j.jctb.2009.04.002. MR2595694

[22] D. Dong. On induced subgraphs of the Hamming graph, arXiv:1912.01780 (2019).

[23] G. Agnarsson, Induced subgraphs of hypercubes, European J. Combin. 34 (2013), no. 2, 155168, DOI 10.1016/j.ejc.2012.09.004. MR2994391

[24] A. Ambainis, X. Sun. New separation between $s(f)$ and bs $(f)$, Electronic Colloquium on Computational Complexity (ECCC), 18 (116) (2011).

[25] Daniel V. Mathews. The sensitivity conjecture, induced subgraphs of cubes, and Clifford algebras, arXiv:1907.12357 (2019).

[26] A. Tikaradze. Induced subgraphs of powers of oriented cycles, arXiv:1908.10463 (2019). 
[27] A. Shadrin, Twelve proofs of the Markov inequality, Approximation theory: a volume dedicated to Borislav Bojanov, Prof. M. Drinov Acad. Publ. House, Sofia, 2004, pp. 233-298. MR2117091

[28] S. Jukna, Boolean function complexity: Advances and frontiers, Algorithms and Combinatorics, vol. 27, Springer, Heidelberg, 2012. MR 2895965

[29] Q. I. Rahman and G. Schmeisser, Analytic theory of polynomials, London Mathematical Society Monographs. New Series, vol. 26, The Clarendon Press, Oxford University Press, Oxford, 2002. MR1954841

[30] K. Regan, Gödel's lost letter and $P=N P$, Tools and Sensitivity, July 2019.

[31] E, Klarreich, Decades-old computer science conjecture solved in two pages, Quanta Magazine, July 2019.

Department of Applied Mathematics, Delhi Technological University, Delhi, India

Email address: fromrohank07@gmail.com

Department of Applied Mathematics, Delhi Technological University, Delhi, India

Department of Applied Mathematics, Delhi Technological University, Delhi, India 